2. M. L. Khandekar, Trends and Changes in Extreme Weather Events: An Assessment with Focus on Alberta and Canadian Prairies, http://www3.gov.ab.ca/env/ climate/docs/TrendsAndChangesIn ExtremeWeatherEvents-April2003.pdf.

Madhav L. Khandekar (mkhandekar@rogers.com) Unionville, Ontario, Canada

\section{Mysteries of the glass transition}

In his intriguing Reference Frame "The Mysterious Glass Transition" (PHYSICS TODAY, February 2007, page 8), James Langer discussed the challenges of glass science. This interdisciplinary field between physics and chemistry has increasingly important applications that now even include the pharmaceutical and food industries.

I like to picture the liquid-glass transition via the following model. Consider a Langevin particle in one dimension moving in an asymmetric double-well potential. The system has a finite relaxation time that diverges as temperature goes to zero, because the relaxation time is related to the barrier to be overcome in the usual Boltzmann expression characterizing rate theory. Consequently, when the system is cooled at a finite rate, it eventually falls out of equilibrium. That process exhibits most of the properties associated with the liquid-glass transition: ${ }^{1-4}$ The liquid-glass transition is gradual rather than sharp, its transition temperature is lower for slower cooling, and the liquid-glass transition is associated with various nonlinear and hysteresis effects.

What happens in the glass transition of the asymmetric double-well potential is that jumps between the two energy minima cease and the system freezes into one minimum or the other. ${ }^{5}$ A glass transition occurs whenever a system doesn't have enough time to equilibrate. Computer simulations confirm that picture for realistic liquids also. The non-Arrhenius behavior usually observed in supercooled liquids is not reflected in the simple model I described but is easily modeled by assuming that the activation energy increases as the temperature decreases.

If that simple model accurately reflects the basics of the liquid-glass transition, then the transition is also just a freezing into an energy minimum. ${ }^{5}$ (Although the distribution of frozen-in energies may deviate from the equilibrium distribution, ${ }^{4}$ it is a minor effect, and to zeroth order the system just freezes configurationally.) Does that eliminate the mystery? Not at all; an enormous challenge still lies in understanding the fairly universal properties of the ultraviscous liquid phase above the glass transition where the viscosity becomes almost $10^{15}$ times larger than that of ambient water. Everything is exceedingly slow in that phase, right? Well, most molecular motion is vibrational, and transitions between different minima are indeed rare. But the diffusion of transverse momentum is actually extremely fast because the exceedingly large kinematic viscosity of the Navier-Stokes equations is the transverse momentum diffusion constant. Thus the ratio between the particle diffusion constant and the transverse-momentum diffusion constant goes from roughly 1 in the less viscous phase to a number of order $10^{-30}$ just above the liquid-glass transition.

Such small dimensionless numbers are rare in condensed-matter physics; they appear to signal that an ultraviscous liquid is more accurately thought of as a solid that "flows." Researchers are not certain, but the existence of a very small dimensionless number characterizing such liquids gives hope that a fairly simple universal theory exists.

\section{References}

1. P. G. Debenedetti, Metastable Liquids: Concepts and Principles, Princeton U. Press, Princeton, NJ (1996).

2. C. A. Angell, K. L. Ngai, G. B. McKenna, P. F. McMillan, S. W. Martin, J. Appl. Phys. 88, 3113 (2000)

3. J. C. Dyre, Phys. Rev. Lett. 58, 792 (1987); J. Bisquert, Am. J. Phys. 73, 735 (2005).

4. J. C. Dyre, Rev. Mod. Phys. 78, 953 (2006).

5. M. Goldstein, J. Chem. Phys. 51, 3728 (1969); F. H. Stillinger, T. A. Weber, Phys. Rev. A 28, 2408 (1983).

Jeppe Dyre (dyre@ruc.dk) Roskilde, Denmark

As someone who has long been interested in the glass transition and glassy-state kinetics, I would like to comment on some of the issues raised by James Langer in his Reference Frame column.

I affirm Langer's statement about healthy contentiousness. Whether or not the glass transition has thermodynamic roots definitely makes for exciting science. The reason some of us think thermodynamics is important is that we find it difficult to dismiss as coincidences the similarities in the values of the kinetic temperature $T_{0}$ and the thermodynamic Kauzmann temperature $T_{\mathrm{K}}$. One common objection to the Kauzmann analysis, that an amorphous solid should not have zero continued on page 72

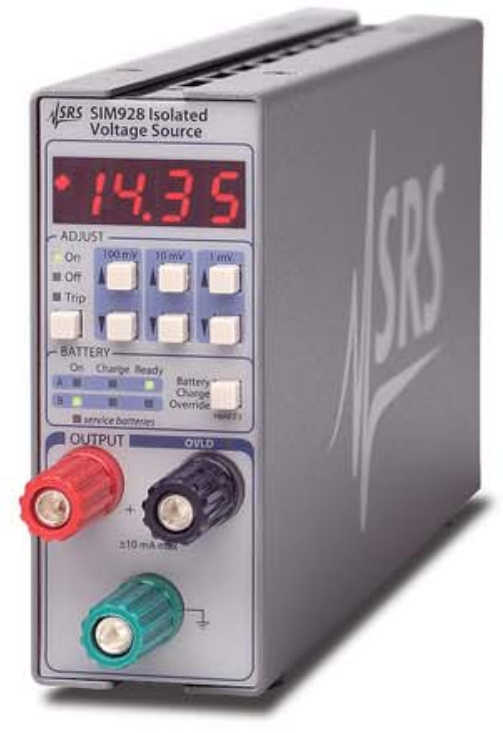

SIM928 ... \$995 (U.S. List)

\section{$\cdot \pm 20 \mathrm{~V}$ isolated voltage source}

- Ultra-low noise output

- Switchable batteries for continuous operation

- Output floats to $\pm 40 \mathrm{~V}$

The SIM928 Isolated Voltage Source is ideal for applications where ultraclean DC voltage is required. Voltage can be set between \pm 20 VDC with millivolt resolution, and the SIM928 delivers up to $\pm 10 \mathrm{~mA}$. The output circuit is optically isolated from all earth-referenced charging cicuitry. As the output battery is depleted, the freshly charged standby battery is switched in to replace it. This provides a continuously uninterrupted isolated bias voltage source.

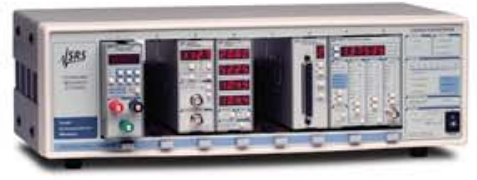

SIM900 Mainframe loaded with a variety of SIM modules See www.pt.ims.ca/16296-9 
entropy, can be assuaged by noting that the entropy at $T_{\mathrm{K}}$ does not have to be zero, just very small.

Langer briefly mentions the success of the simplistic Adam-Gibbs (AG) model in describing the dynamics of supercooled liquids. Its nonlinear extension into the glass-transition region and glassy state (NLAG) is also surprisingly successful. ${ }^{1}$ That extension is based on concepts introduced by several researchers over several decades: Simon Rekhson in 1994, George Scherer in 1984, Cornelius Moynihan in 1976, O. S. Narayanaswamy in 1971, and others. The successes of the NLAG model go far beyond expectations, and raise issues of their own. The resolution of these issues might provide important clues to a theoretical understanding of the glass transition.

- As noted by Langer, the experimentally observed effective activation energy $E(T)$ increases rapidly with decreasing temperature down to the glass-transition temperature $T_{\mathrm{g}}$, but it then decreases through the $T_{\mathrm{g}}$ range until it reaches a constant value $E\left(T_{\mathrm{g}}\right)$, so that glassy-state relaxation exhibits Arrhenius behavior. The singularity at $T_{0}$ noted by Langer only occurs in the equilibrium supercooled liquid state and not in the experimentally observed nonequilibrium glassy state. The change from nonArrhenius to Arrhenius behavior at $T_{\mathrm{g}}$ is well described by the NLAG model and its precursor, the Tool-NarayanaswamyMoynihan model.

- NLAG predicts a simple relation between the ratio $T_{K} / T_{\mathrm{g}}$ and an empirical constant that parameterizes the nonlinearity of the glass transition and glassystate kinetics. This intriguing prediction needs to be independently confirmed or unambiguously refuted.

- The NLAG model, together with the plausible assumption that smaller localized activation energies $\Delta \mu$ enable the kinetic $T_{\mathrm{g}}$ to get closer to the thermodynamic $T_{\mathrm{K}}$, generates many of the correlations captured by Angell's fragility. In fact, the ratio $T_{\mathrm{K}} / T_{\mathrm{g}}$ is an excellent metric that allows fragility to be applied to the glassy state.

- Estimated values of $\Delta \mu$ for canonical glasses are often comparable with rotational energy barriers in polymers, and ionic, covalent, and hydrogen bond strengths. In these cases the NLAG model is almost quantitatively accurate. - Incorporation of a distribution in $\Delta \mu$ yields a respectable account ${ }^{2,3}$ of ther- mal manifestations of motions in hydrated proteins and B-DNA. ${ }^{2,4}$ The mean value for $\Delta \mu$ is comparable with hydrogen bond strengths, albeit with a large uncertainty, and the large standard deviation $-30 \%$ of the average - is consistent with the insightful but qualitative analysis of Jennifer Green and coworkers. ${ }^{4}$ The fact that NLAG gives a decent account of annealing in hydrated proteins and B-DNA strongly supports Austen Angell's suggestion that the glass transition and protein dynamics have much in common. ${ }^{5}$

I share Langer's belief that shortrange interactions are probably the key. Since the current models accommodate a wide range of interactions, such as covalent, hydrogen, and ionic bonding, the glass-transition phenomenon is evidently insensitive to the details of those interactions. This generality is missing from too many theoretical attempts at explaining the problem. Perhaps the averaging of details is why the simplistic NLAG model is so successful.

\section{References}

1. G. W. Scherer, J. Amer. Ceram. Soc. 67, 504 (1984); I. M. Hodge, Macromolecules 20, 2897 (1987).

2. G. Sartor, E. Mayer, G. P. Johari, Biophys. J. 66, 249 (1994).

3. I. M. Hodge, Biophys. J. 91, 993 (2006).

4. J. L. Green, J. Fan, C. A. Angell, J. Phys. Chem. 98, 13780 (1994).

5. C. A. Angell, Science 267, 1924 (1995).

Ian Hodge

(ian.hodge@rit.edu)

Rochester Institute of Technology Rochester, New York

I do not see any mystery in James Langer's "mysterious glass transition," at least with respect to inorganic glasses of one-component systems like silicon dioxide, boron oxide, and so on. To understand the glass transition of inorganic materials, one first has to understand why crystals melt. Near the melting temperature, electrons occupy more and more excited states as temperatures increase. Electrons in excited states possess wavefunctions different from those in their low-energy or ground states. Different wavefunctions mean that the probability distribution of the electrons in space changes. The core ions will be driven to new places as they interact with the excited electrons. However, the electrons will change again and again to other states with different wavefunctions. The arbitrary time series of sufficient electrons in their excited states will cause the core ions to continuously change position. That scenario corresponds to a melt.

As the melt cools, the electrons will occupy more and more low-energy states. If the forces of the electrons in their low-energy states are not strong enough to induce a regular order of the core ions, the transition to a glass occurs. Thus melting of chemically bonded solids, and glass formation from their melts, is basically an electronic effect generally neglected in publications dealing with properties of melts and glasses. ${ }^{1}$ Glass formation from the melt depends on the strength and sufficiently large number of directed bonds (to stabilize the noncrystalline order) and on the melting entropy $\Delta S_{m}$ (that is, melting enthalpy $\Delta H_{m}$ divided by melting temperature $\left.T_{m}\right)$. If $\Delta S_{m}$ is small, only a little entropy is released and produced once a bond closes, and the temperature increases locally by just a small amount. This implies that neighboring directed bonds of the undercooled melt can be broken only within a relatively small temperature range below $T_{m}$. This interval has to be passed fast enough for glass formation. If $\Delta S_{m}$ is large, the temperature interval of recalescence is relatively large to reach $T_{m}$ and the undercooled melt has enough time to rearrange to crystals during cooling. ${ }^{2}$

Now it is easy to understand the "mystery" of the glass transition or what occurs in the glass-transition range. (Imagine that the temperature is rising.) In that range, bonding electrons start to occupy excited states. This causes an additional mechanism for the thermal expansion, an additional contribution of the specific heat capacities (not causing a "jump," however), and an increase of the damping of resonances of many kinds in glasses. The worldwide standard procedure to determine $T_{g}$ in glass science is based on the change of the slope of dilatometer curves, not mentioned by Langer. As a consequence of the scenario described here, there is no phase transition at $T_{g^{\prime}}$ just an exponential freezing out of electrons from higher to lower energy levels with decreasing temperature.

\section{References}

1. H.-J. Hoffmann, Phys. Chem. Glasses 45, 227 (2004).

2. H.-J. Hoffmann, Phys. Chem. Glasses 46, 570 (2005)

Hans-Jürgen Hoffmann
(hoffmann.glas@tu-berlin.de)
Technical University of Berlin
Berlin, Germany

With the very noncrystalline nature of the state labeled "glass," the use of terms like "lattice sites" by some physicists is misleading if not erroneous. Having spent nearly 40 years researching solid-state chemistry using diffraction 


\section{MAENETS}

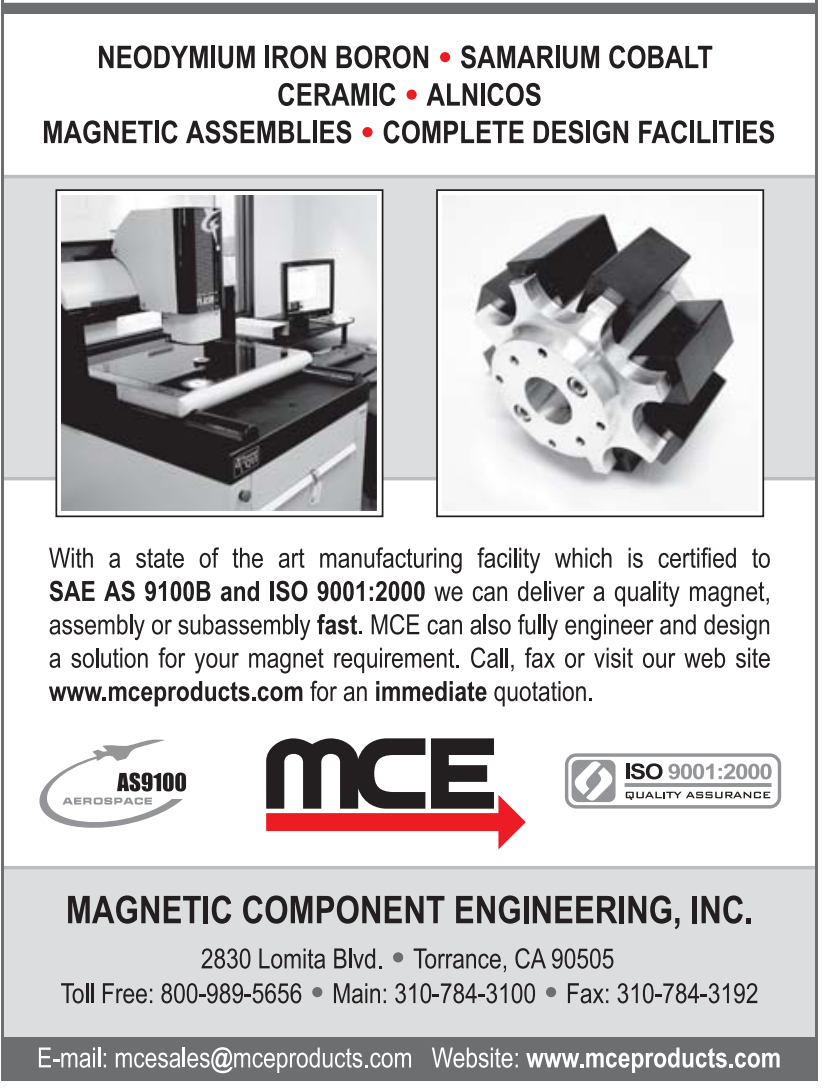

See www.pt.ims.ca/16296-32

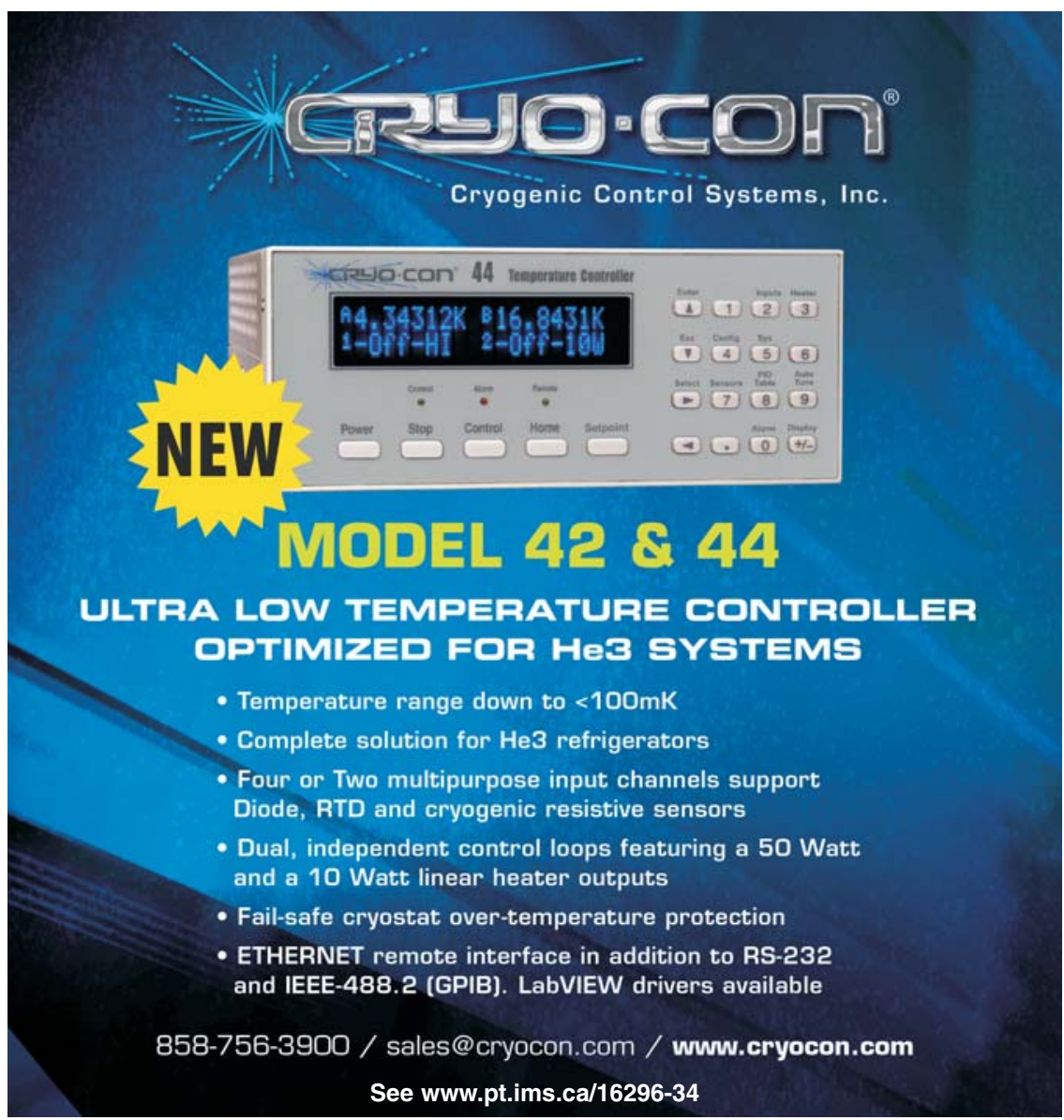

methods, I can say that the glass state is not just limited to glass - that amorphous state of polymeric silicon oxide with doping of other oxides, including boron oxide. In fact, all polymers, including those extensively used in daily life starting with organic monomers, show the "mysterious" glass transition.

A study of the glass transition in any polymeric material is necessarily dictated by complex variations in the motions of the polymeric chain segments, which form as sheets, coils, helices, and the like. The glass transition in the case of doped silicon oxides may be ascribed to the conformational changes in the vicinity of the tetrahedral silicon, while in polymers it involves oxygen atoms in the polymeric helices or sheets.

One can draw inferences from the crystal structures of pure silicon oxides such as quartz in that even those crystals enter the glass state upon heating. ${ }^{1}$ Then it is very difficult to recover the original crystal with the same characteristics.

When melted, even crystals of sucrose, a simple everyday compound, lead to a glassy state that is far more mysterious than the glass itself.

To understand the underlying principles of the behavior of the glass state, we must use radial distribution functions from diffraction data to study these mysterious glass transitions, particularly in regard to their structural details at the molecular level.

\section{Reference}

1. B. D. Sharma, J. Chem. Educ. 64, 404 (1987).

Brahama D. Sharma (mercury610@aol.com) Pismo Beach, California

Many thanks to the authors for their comments about my Reference Frame column. I have just a few remarks in reply.

Ian Hodge and I agree that we still need a deep, first-principles understanding of the remarkably successful Adam-Gibbs formula, in both its original and extended nonlinear versions. It will be interesting to see whether the physical mechanisms underlying the two related phenomenologies are actually the same in their respective regions of validity.

Jeppe Dyre remarks that some basic features of the glass transition are captured by the simple asymmetric doublewell model. He clearly understands that there is a great deal more to the mystery than that, and I think he is making his point in an interesting way. Viscous relaxation rates near the glass transition are about 15 orders of magnitude slower than molecular vibration 
frequencies. As Dyre points out, processes on both time scales are taking place in glass-forming systems. How do we relate one to the other?

I admit I'm puzzled by the other two letters. True, all molecular interactions are ultimately quantum mechanical in nature; but, like most specialists in this field, I see no reason to think that the generic glass transition is intrinsically a quantum, as opposed to classical, phenomenon. Hans-Jürgen Hoffmann seems to imply otherwise. In reply to Brahama Sharma, perhaps it will be helpful to say that when I talk about the "glass transition," I am thinking of metallic glasses, polymeric glasses, and a wide variety of other noncrystalline materials, not just silicate glass.

James Langer

(langer@physics.ucsb.edu) University of California, Santa Barbara

\section{Sound}

\section{commentary}

I'm tickled to see that the feature article "Medical Diagnostic Ultrasound" (PHysics TODAY, March 2007, page 44) is by my neighbor, Carr Everbach, and I enjoyed it. He starts by mentioning "sounding" water depths from the sound given by a lead weight hitting the bottom; he also mentions "the propagation time" and that the phrase "to sound something out" is connected.

I'm puzzled, though, since the sound of the sounding lead hitting the soft, muddy Mediterranean Sea bottom would hardly be heard in the air, given the acoustic mismatch. Is there evidence that stethoscopes were held against the ship's hull? Or did someone perhaps press an ear against the hull?

The online edition of the Oxford English Dictionary says that "sound" in this meaning is simply related to water, alas, and not to aural sound. Moreover, the speed of sound in water is so high that the tiny propagation-time interval for sound to travel from the sea bottom is hard to discern. Maybe that interval was the fall time in water, a viscous medium?

Perhaps Carr has other evidence, so I may sleep soundly about this.

\section{Leonard Finegold} (L@drexel.edu)

Drexel University Philadelphia, Pennsylvania

Everbach replies: Leonard Finegold's points are sound on several counts. The "sounding machine" used by the ancient Greeks and for thousands of years thereafter consisted of a lead weight that was thrown overboard, into the sound, tied to a knotted rope. The "propagation time" was the time for the weight to fall at its terminal velocity to the sea bottom; that time was proportional to the length of rope paid out-that is, distance from the source. Thanks for sounding me out on this question, Len!

E. Carr Everbach (ceverba1@swarthmore.edu) Swarthmore College Swarthmore, Pennsylvania

\section{Professional kindnesses}

In harkening back to life in physics 60 or more years ago, it is useful to look at the American Astronomical Society, whose membership today is about what American Physical Society membership was then.

Chatting recently with an astronomer friend, I was interested to learn that he regularly receives complimentary preprints or reprints from colleagues doing similar research. I cannot recall from my personal experience enjoying that particular form of collegial exchange, but it reminds me of a time when professional relationships were more personal, more cordial, and less competitive.

Preprint exchange strikes me as a social amenity that should be encouraged today to foster friendly personal and professional relationships. It should be a general practice to send preprints or reprints to anyone who has made a significant contribution to one's work and is mentioned, or should have been mentioned, in the acknowledgments.

Lawrence Cranberg (info@lawrencecranberg.org) Austin, Texas

\section{Correction}

November 2007, page 76-The Physical Science Study Committee was mistakenly referred to as the Physics Science Study Committee.

\section{Rights \& Permissions}

You may make single copies of articles or departments for private use or for research. Authorization does not extend to systematic or multiple reproduction, to copying for promotional purposes, to electronic storage or distribution (including on the Web), or to republication in any form. In all such cases, you must obtain specific, written permission from the American Institute of Physics.

$$
\text { Contact the }
$$

AIP Rights and Permissions Office, Suite $1 \mathrm{NO1}$

2 Huntington Quadrangle,

Melville, NY $11747-4502$

Fax: 516-575-2450

Telephone: 516-576-2268

E-mail: rights@aip.org

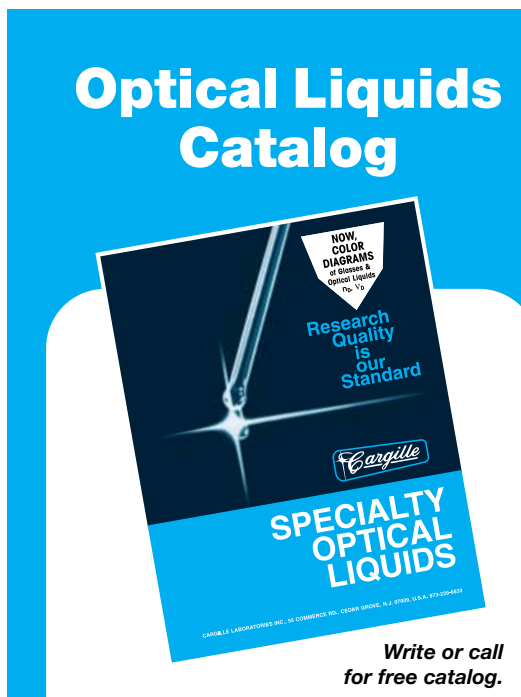

New catalog of specialty optical liquids features high-transmission, safe-handling, laser liquids, plus fused silica matching liquids, and specific refractive index liquids (1.300-2.11 $\left.n_{D}\right)$. Now includes comparative diagrams of glasses and optical liquids.

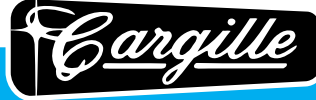

Cargille Laboratories Inc.

55 Commerce Road, Cedar Grove, NJ 07009 973-239-6633 • Fax 973-239-6096 cargillelabs@aol.com • www.cargille.com

See www.pt.ims.ca/16296-33

WI N \$3000

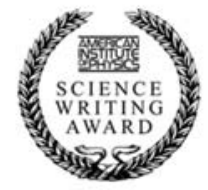

A CALL FOR ENTRIES

\section{8}

American Institute of Physics Science Writing Award in Physics and Astronomy

To be awarded to a professional journalist for articles, radio, or television programs dealing with physics or astronomy and intended for the general public.

Entries must have been published between January 1, 2006 and December 31, 2007 and written or translated into English.

\section{Deadline: March 1, 2008}

For an entry form or more information, visit www.aip.org/aip/writing

or contact Tatiana Bonilla, Media \& Government Relations American Institute of Physics Tel: (301) 209-3096

Email: tbonilla@aip.org 